\title{
The Construction Mode of the Sadness Experience of Losing-Single-Child Groups under Chinese Cultural Background
}

\author{
Xinliang Wang and Chao Wu \\ (1.Lanzhou Institute of industry, Lanzhou, Gansu 730050) \\ (2.Gansu National Normal College, Hezuo, Gansu 747000)
}

Keywords: Chinese culture; Losing-single-child groups; Sadness experience; Mode construction

\begin{abstract}
The loss of only child events have a great influence on the life and work of the relevant parties and helping them to get out of their grief and return to their daily life is an urgent task for the development of contemporary Chinese society. Based on the background of China's cultural development, this paper analyzes the process of the sadness experience of the people who have lost their only child and the characteristics that are shown and puts forward the mode of constructing their sadness experience.

There are many studies on the sadness experience of losing-single-child groups abroad; In contrast; most of our research is based on foreign research; The experience process of losing single child lasts longer and more complex than other bereavement experience process;

More attention should be paid to these groups of people. Based on the background of China's cultural development; this paper puts forward the mode of constructing their sadness experience and help them to get out of the misunderstanding of their sadness experience and promote their healthy development;
\end{abstract}

\section{The Experience Process of Losing Single Child Groups}

Sadness is a kind of feeling that is complex and difficult to be understood by others. Losing single child sadness is the most special and serious experience among all the sadness experience and should be taken seriously. Combining with the background of China's cultural development, losing single child sadness experience is divided into five important stages. It is noteworthy there are individualized differences between experience forms that are shown in the course of losing single child sadness experience and duration due to the individual difference in its specific development. Some of the performance may run through the whole process of development and It is difficult to retreat for a period of time. For those who lose their single child, it is difficult to get out of the predicament of losing their single child experience, and it is difficult to get out of the sad situation. The specific experience of losing single child can be divided into:

The first stage: consternation, sadness and refusal, repeated recollection stage. This stage refers to the consternation and sadness the related parties express when they first hear the news of his only child's death. Most people who lose their single child refuse to eat and drink water, with tears all day long. More serious ones have physical syncopes which cause intermittent pauses in thinking. For the first reaction to the loss of the only child, they usually show a rejection. That is because the event happens suddenly and they are not prepared for psychological preparation. The behavior of the ones who lose a single child are completely different from the ones whose children are dead due to a long-term disease. This is because they are less amazed as they are already prepared in advance. The overall reflection is slightly smaller after a psychological stress trauma. At this stage, the ones who lose a single child will consciously reject the occurrence of this event and even have an illusion. There are some children's life scenes in their brains and they often recall the past scenes with the child fantasizing that the child will come back one day. The scene in everyday life also makes them think of their own child causing another pain. The duration of this stage is at least half a year or so or even longer of 1 or 2 years.

The second stage: anger, resentment and blame. At this stage, the ones who lose a single child will enter the stage of anger and complaining and start to have the mind of blame lasting 3 to 5 
years. At this stage they begin to neglect the behavior of their partner and often feel angry or have the mind of complaining and more serious ones will have the mind and behavior of revenge for the perpetrator. In addition they will have some extreme ideas and even hope the earth is destroyed or use alcoholism to paralyze themselves.

The third stage: numbness, depression and escape. At this stage, the ones who lose a single child confirms that the thing has happened and cannot be redeemed and anything they do does not work. They will have a long term mind of numbness and continue to be low-spirited and cannot control your own behavior and are out of control. Some of them will have the mind of escape and avoid talking about a child or a topic about the loss of a single child and unwilling to participate in social activities and more serious ones have the mind of depression. Some of them will have suicidal thoughts and behavior. This stage lasts 2 to 3 years or even longer.

The fourth stage: sobriety, remorse and resistance. At this stage, the ones who lose a single child accept the fact that the child is dead but have the mind of hoping to exchange their own life with the life of the dead child. Under this sense of consciousness, they gradually accept the fact of the child's death, and begin to think calmly about the next problem, the problem of no successors. They will consider the relevant one child policy and pay more attention to the family planning policies and hope the harm caused by the loss of a single child could be shared with the help or assistance of the government.

The fifth stage: acceptance and return. At this stage, the ones who lose a single child can accept the fact of the child's death and begin to realize that their future life will continue. This process will take place five years after the loss of a single child. They do so for the child's wish or the awakening of their consciousness begin to return to daily life and gradually get out of the loss of a single child. The ones who lose a single child begin to realize that the child's death is not his own fault and begin to explore and think about the future, and hope that children in heaven can see their parents very happy. The ones who lose a single child begin to gradually get out of the dilemma through their own conscious mental regulation and with the help of social workers

\section{The Characteristics of the Sadness Experience of Losing a Single Child}

Firstly, the sadness experience of losing a single child is the greatest sadness that the ones losing a single child can experience. For the sad situation in life, the bereaved life is an inevitable experience, which will give the bereaved great emotional pains. For the ones losing a single child losing a child is the greatest hurt to them, and it can be regarded as the greatest sorrow of life, which will arouse the grief of the parents. Less serious ones may have the symptoms of restlessness or loss of appetite and serious ones would have the mind of depression or the suicidal thoughts and even more serious ones would have negative impact-revenge on society。

Secondly, the degree of the sadness experience of losing a single child is Influenced by a variety of factors mainly including personality characteristics of the ones losing a single child or the cause of the child's death, the emotional connection state between them and children. Specifically, it can be distinguished by the context of the event and the ones losing a single child can be helped out to find the way of emotional relief.

Thirdly, the sadness experience of losing a single child is Influenced by emotion. Emotions influence the normal functioning of individual functions to some certain extent and have great regulation effect on interpersonal relation as well. On a certain level, Sadness can gain sympathy from others and anger can mobilize positive resources to protect oneself. The ones losing a single child are influenced by sadness for a long time and strand in extreme emotions. It is easy for them to have morbid or other complex mind. This is because the ones losing a single child see the missing of the child as the whole of life. It is difficult for them to get out of the sad situation. Most of them are immersed in the yearning for the child, and can not extricate themselves for a long time or adopt negative escape. Therefore, for the losing-single-child one's emotional relief, the specific analysis is used for the specific case and the specific situation.

Lastly, sadness experience of losing a single child is accompanied by negative emotions. 
Negative emotions or social cognition will produce negative psychological hints for the ones losing a single child. The long-term acceptance of such negative psychological hints can easily lead the ones losing a single child into a relatively negative state and thus reduce their independence and let them down. sadness experience of losing a single child is often accompanied by negative emotions commonly including helpless sadness, depressive sadness, remorseful sadness, avoidant and resentful sadness, etc.

\section{The Mode of Constructing the Sadness Experience of the Losing-Single-Child Groups}

Increasing policy input and building the perfect compensation mode of the sadness experience of the losing-single-child groups.

The construction of this mode relies mainly on government policy support and help losing-single-child groups to get rid of the sad experience as soon as possible through adopting particular policy aids. In terms of specific methods, support funds can be increased and many policies can be introduced for losing-single-child families and the community-based service for their aged life should be increased and their needs should be met in policy. Besides, the government should pay attention to providing more subsidies for the special families carrying out family planning and make the receiving policy more flexible and improve the social welfare system for losing-single-child groups and help build relevant functional departments managing losing-single-child groups through laws and multisectoral coordination. The needs of losing-single-child groups are met and the compensation mode of the sadness experience of losing-single-child groups is established and improved through govermental policies.

Increasing social activities and organizing and establishing social workers service mode of the sadness experience of losing-single-child groups.

The activities of social groups can shorten the time of the sadness experience of losing-single-child groups and help build the confidence of continuing life. In the losing-single-child activities of registration of social groups, the participation of non professional volunteers should be stressed. Most of the help for losing-single-child groups becomes formalistic due to non professional volunteers lacking formal training. Investment should be enhanced for them. When hiring social workers, the government should increase hiring the social workers with certain expertise and establish professional social workers service talent teams and conduct interventional services and play an important role in social workers service. On this basis, various forms of social activities can be widely carried out and establish and improve social workers service mode of the sadness experience of losing-single-child groups and make them form a positive and optimistic attitude.

Strengthening the guidance of correct ideas and establishing informal care mode of the sadness experience of losing-single-child groups.

Informal care refers to the care and concerns from the relatives, friends, colleagues, or neighbors close to the losing-single-child one. This is because they have a close relationship with the losing-single-child one and understand what happens early and the specific requirements of the losing-single-child one and can give a hand in time under proper circumstances, which makes them feel .

human warmth and reduces the probability of negative and extreme behavior. This group of people are regarded as informal organization and the care they give to the losing-single-child one is called informal care. That is because it is not formally registered for management. There is no clear rules and regulations which can be used or the resources for action are limited. Generally its specific activities rely mainly on the platform of the community and there is a limited range of activities, belonging to a small range of activities. The organizational form these informal organizations take gather losing-single-child families together and carry out the collective activities. The collective way has some danger because it is not guided by right ideas and it is easy to go astray in the development. This requires that the correct concept should be established, and the correct development orientation should be formed. Group activities can be classified and organized and losing-single-child groups who have the same situation can participate in the same one. Besides, 
The community should also establish a perfect and effective service system and understand the actual situation of these informal care activities in time. and exchange with them for the activities and give full support to these informal organizations

\section{Conclusion}

In the development of modern society, losing-single-child groups, as a special group, the overall care for whom should be strengthened. The sadness experience process of losing single child lasts longer and more complex than other bereavement experience process. More attention should be paid to these groups of people. Based on the background of China's cultural development, this paper puts forward the mode of constructing their sadness experience and help them to get out of the misunderstanding of their sadness experience and promote their healthy development. Besides, the sadness experience of losing-single-child groups as an emotional and lasting experience process is inevitable. losing-single-child one's emotional relief should be increased after an event happens and a variety of modes should be constructed actively.

\section{References}

[1]Xiu, L, 2014. A study on the mode of losing-single-child people's grief adjustment and its localization intervention. Nanjing University of Chinese Medicine

[2]RICE PL, 2000. Health Psychology. China Light Industry Press. PP. 145-46

[3]Tingting, Wang. The survey shows: $20 \%$ parents lost their feelings and even divorced. Legal Evening News. 2014-07-10

[4]Tao,M and Jing,L 29018 Losing-single-child parents' aged life in Jiangsu province is faced with multiple governance. Jiangsu network, China. 2014-01-20

[5]Lishuang, Y. Losing-single-child family needs old-age security. Beijing Daily. 2014- 01- 15

[6]Zhenhua,L. 2014. Reflection and reconstruction of the family assistance system of " losing-single-child family " in China. GUANGXI Social Sciences. (7):156- 161.

[7]Yong,W.2014. Thinking about the construction of the social support system of losing-single-child family. Population and family planning. (5):24- 25.

[8]Qiangling,S. 2014. The problem of losing-single-child people's aged life and the Countermeasures under the view of aging. Chinese Journal of Gerontology. 34(15):403- 405.

[9]En,C. .2014.Reconstructing the social support network: A discussion on the self-organization formation mechanism of losing-single-child group -- based on two cases in Shanghai. Journal of Nanjing Institute of Technology (SOCIAL SCIENCE EDITION) 02:14-15.

[10]Chunlin,X.2015. A preliminary study of the Chinese mode of sadness and comfort. Journal of Jiangxi Normal University philosophy and Social Science Edition. (2):96-98.

\section{Brief introduction about the author}

Xinliang, Wang (1988-), Female (Han), born in Gansu silver, master, lecturer, main research direction: mental health education, psychological consultation.

Chao,W (1981- ), Female (Han), born in Anyang, Henan, master, lecturer, main research direction: student psychology 